\title{
Opiatintoxikation - Das sollten Sie wissen für die Ergänzungsprüfung
}

Rico Kuhnke, Wolfgang von Meißner
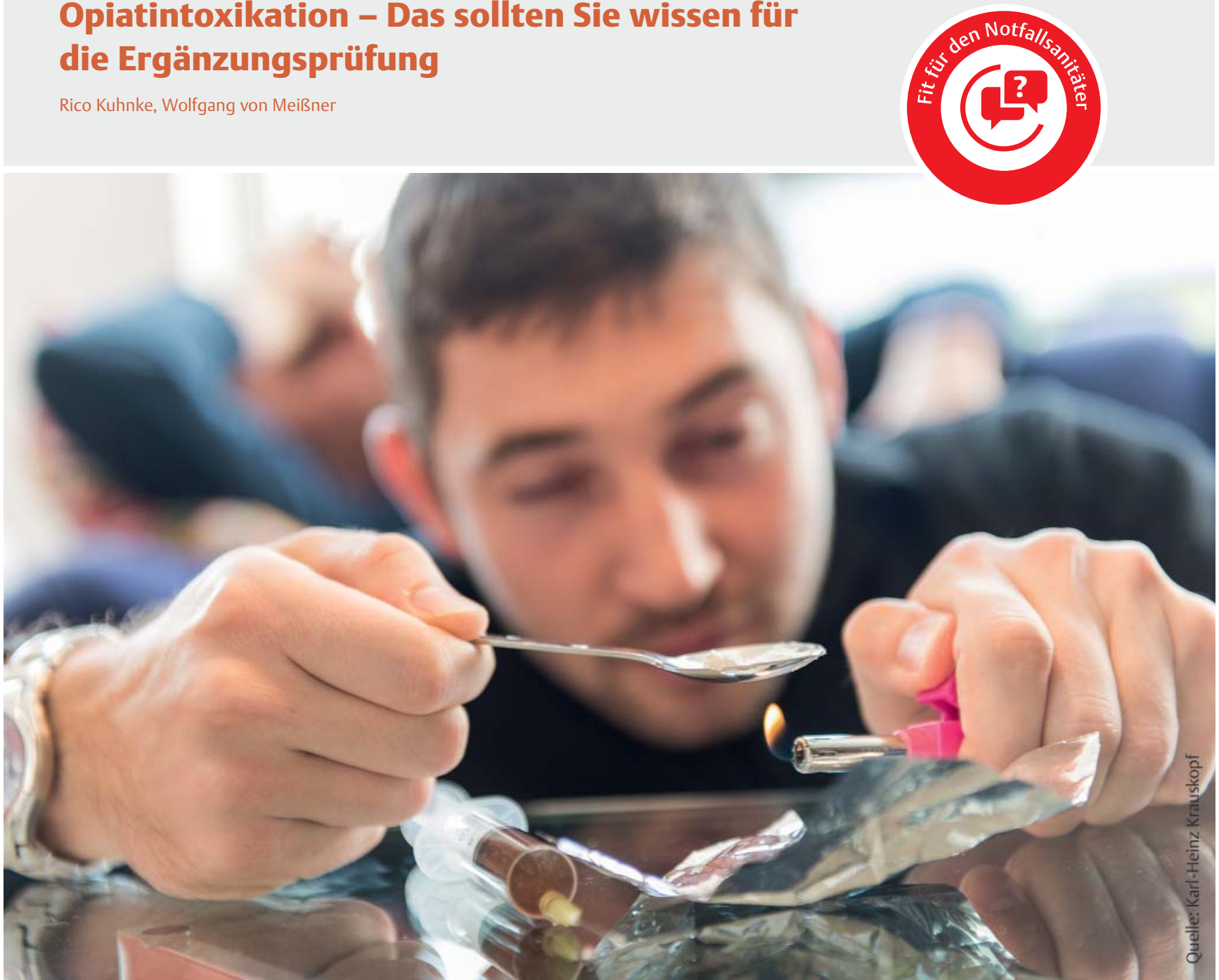

retten! macht Sie fit für den Notfallsanitäter: In jeder Ausgabe arbeiten wir anhand eines Fallbeispiels einen interessanten Einsatz algorithmenkonform auf. Anhand von exemplarischen Fragen zu erweiterten Notfallmaßnahmen, Kommunikation und Rahmenbedingungen können Sie sich auf die Ergänzungsprüfung vorbereiten - egal, in welchem Bundesland Sie arbeiten.

\section{Fallbeispiel}

\section{Einsatzmeldung}

„Hilfelose Person, Hauptbahnhof, im Bereich der Damentoiletten; die Polizei wurde mitalarmiert und ist auf der Anfahrt." Bereits im RTW zieht sich die Beifahrerin ein 2. Paar Einmalhandschuhe über und legt ihrem Kollegen ein weiteres Paar in die Ablage. Wie üblich trägt das Team Schutzbrillen. Am Haupteingang werden die Rettungskräfte von einem jungen Mann erwartet, der sie zum Toilettenbereich führt. Das Team hat das Einsatzfahrzeug abgeschlossen und auf die Mitnahme der Fahrtrage verzichtet, da zwischen den Toiletten und der Anfahrt mehrere Treppenabsätze liegen.

\section{Situation vor Ort}

Am Einsatzort angekommen, finden die Rettungskräfte eine sehr schlanke, ca. 40-jährige Frau bewusstlos auf dem Boden vor. Auf den Fliesen verteilt liegen die typischen Fixerutensilien. Neben einer leeren Insulinspritze mit aufgesteckter Nadel befinden sich ein Feuerzeug, eine Tüte mit weißem Pulver sowie ein gebrauchter Löffel und ein offenes Aluminiumpäckchen ohne Inhalt. Vorsichtig schiebt die Notfallsanitäterin die Utensilien mit dem Schuh in die Ecke. Da die Räumlichkeiten sehr beengt sind, entscheidet sich das Team, die bewusstlose Patientin mit dem Rettungsgriff nach Rautek in den Vorraum zu bringen. Während die Notfallsanitäterin die Patientin vom Boden aufnimmt, breitet ihr Kollege die 
mitgebrachte Einmaldecke auf den kalten Fliesen aus. Die leichtbekleidete Frau wirkt auf den ersten Blick, als käme Sie aus dem Strichermilieu. Ganz in der Nähe befindet sich der Straßenstrich und die Toiletten werden immer wieder für den Konsum von Drogen genutzt.

\section{Sicherung der Vitalfunktionen}

Bereits beim Umlagern fällt auf, dass die Patientin ohne Muskelspannung und völlig schlaff in den Armen liegt. Im Licht des Vorraums wirkt sie auffallend blass und zyanotisch. Kaum auf dem Boden abgelegt, beginnt die Kollegin mit der Sicherung der Vitalfunktionen nach dem standardisierten ABCDE-Schema. Parallel organisiert ihr Kollege das Material und öffnet den Notfallrucksack.

\section{A-irway}

Die Atemwege sind sichtlich verlegt. Aus dem rechten Mundwinkel fließt Speichel. Noch bevor die Notfallsanitäterin den Kopf überstreckt, dreht sie diesen vorsichtig zur Seite und saugt die oberen Atemwege mit einem großlumigen Absaugkatheter ab. Dann führt sie den Kopf nach hinten, und die Patientin atmet hörbar spontan ein.

\section{B-reathing}

Die Atemfrequenz ist mit einer Frequenz von 6/min deutlich verlangsamt und nicht ausreichend. Das Team entscheidet sich zu einer assistierten Beatmung: Der Kollege übernimmt diese mit dem vorbereiteten Beatmungsbeutel mit Reservoir und Sauerstoff, während die Kollegin mit der Untersuchung fortfährt. Die zuvor angelegte Pulsoxymetrie zeigt keinen Wert. Die Finger der Patientin sind kühl und nur schlecht durchblutet.

\section{Konflikt mit der Begleitperson}

Der junge Mann, der das Team zur Patientin geführt hat, ist sichtlich nervös und beobachtet das Rettungsdienstteam während der Versorgung. Auf Nachfragen zur Situation gibt er nur vage Antworten. Der Kopfhelfer beobachtet aus dem Augenwinkel, wie sich der Mann für das Ampullarium interessiert. Als dieser die Hand ausstreckt, reagiert er sofort. Sehr bestimmt wendet er sich an den jungen Mann: „Hände weg! Die Medikamente werden für die Therapie benötigt. Bitte gehen Sie einige Schritte zurück und stellen Sie sich in die Ecke!“ Der Angesprochene ist sichtlich erschreckt, befolgt die Anweisung aber unwillig. Dabei murmelt er etwas Unverständliches.

\section{C-irculation}

In der Zwischenzeit hat die Notfallsanitäterin den peripheren Puls an der A. radialis gefühlt. Dieser ist zwar nur schwach tastbar, aber regelmäßig, und mit einer Frequenz von 90/min akzeptabel. Auf die Messung des Blutdrucks verzichtet das Team und legt für die weitere Überwachung ein EKG an. Wie zu erwarten, zeigt das EKG einen unauffälligen Sinusrhythmus.

\section{D-isability}

Aufgrund der Umstände prüft die Notfallsanitäterin den Pupillenstatus der Patientin. Die Pupillen sind isokor und maximal verengt, auf Licht reagieren sie kaum. Der Befund bestätigt den Verdacht auf eine Heroinintoxikation als Grund für die Bewusstlosigkeit. Da sich die Atmung nicht stabilisiert, entscheidet sich das Team, schnellstmöglich Naloxon i. v. zu geben.

Das Legen eines peripher-venösen Zugangs bereitet große Mühe. Am rechten Unterarm findet sich ein kleineres Gefäß; dort kann die Notfallsanitäterin eine rosa Venenverweilkanüle (ID 0,8 mm, 20 G) etablieren. Die Blutzuckermessung ergibt einen Wert von $78 \mathrm{mg} / \mathrm{dl}$.

\section{E-xposure}

Vor der Applikation des Antidots vervollständigt die Notfallsanitäterin ihre Diagnose mit einer kurzen körperlichen Inspektion der Patientin. Strukturiert arbeitet sie sich vom Kopf bis zu den Füßen vor. Hinweise für Verletzungen sind keine zu finden. An verschiedenen Stellen lassen sich Einstiche unterschiedlichen Alters ausmachen. Die eine oder andere Einstichstelle ist entzündlich verändert.

\section{SAMPLER+S-Schema}

Eine gezielte SAMPLER+S-Anamnese lässt sich nicht durchführen. Bis auf die Untersuchungsergebnisse kann das Team keine zusätzlichen Informationen gewinnen. Der anwesende junge Mann gibt sich als Freund der Patientin zu erkennen; seine Angaben zu Allergien, Medikamenten und Patientengeschichte sind aber nicht zu verwerten. Lediglich die Frage nach der letzten Mahlzeit kann der Freund beantworten: Er gibt an, dass sie beide noch nichts gegessen hätten und im Anschluss in ein Café gehen wollten. Der junge Mann wirkt nun zunehmend unruhig, er zittert und schwitzt.

\section{Eintreffen der Polizei}

Als die Polizeibeamten eintreffen, nutzt der Freund der Patientin den Augenblick der Übergabe und stürzt aus der Eingangstür. Dabei schubst er einen Beamten, der unsanft zu Boden fällt. Die Polizisten nehmen sofort die Verfolgung auf. Nach kurzer Zeit kehren sie zurück; sie haben den Mann im Menschengedränge verloren und bieten nun ihre Hilfe an. Ein Beamter wird gebeten, die Infusion zu halten, der andere soll den nachgeforderten Notarzt einweisen und möglichst noch eine zusätzliche Decke aus dem Fahrzeug bringen.

\section{Antidot-Gabe}

Die Notfallsanitäterin hat in der Zwischenzeit 1 Ampulle Narcanti $^{\circledR}$ (Naloxon 0,4 mg) auf $8 \mathrm{ml}$ mit $\mathrm{NaCl} 0,9 \%$ verdünnt und verabreicht diese nun langsam und in Schritten von $1 \mathrm{ml}$ (=0,05 mg Naloxon). Den Kopfhelfer weist sie an, die Atmung der Patientin aufmerksam zu beob- 
achten. Nach der Gabe von 0,3 mg Naloxon hat sich ihre Atmung bei einer Frequenz von 14/min nahezu stabilisiert. Mittlerweile hat auch die Pulsoxymetrie eine Anzeige: Die Sauerstoffsättigung ist mit $95 \%$ akzeptabel. Das Team beendet die assistierte Beatmung und bereitet alles für einen Transport ins Fahrzeug vor.

\section{Ermittlung der Identität}

Das Notarztteam ist mittlerweile eingetroffen und hat den Rettungskräften geholfen, die Patientin in das Fahrzeug zu bringen. Die Identität der Patientin konnte die Polizei mithilfe des Ausweises ermitteln. Die Beamten benötigen für ihr Protokoll nun noch nähere Informationen zur Verdachtsdiagnose. Als sie die Notfallsanitäterin dazu befragen, verweist diese auf ihre Schweigepflicht.

\section{Der Algorithmus}

Das Team nutzt zur Bearbeitung des beschriebenen Falls den Algorithmus „Opiatintoxikation - Naloxon“ der Deutschen Angestellten-Akademie (DAA) Meiningen ( $\triangleright$ Abb. 1), die ihre Algorithmen anhand der Musteralgorithmen des Deutschen Berufsverbands Rettungsdienst (DBRD) erstellt hat. Die Unterschiede zwischen dem Algorithmus der DAA und dem des DBRD sind zu vernachlässigen und beschränken sich im Wesentlichen auf das Design.

\section{PRAXISTIPP}

Sollten Sie in Ihrem Rettungsdienstbereich ein abweichendes Konzept nutzen, arbeiten Sie die Unterschiede heraus. Überlegen Sie sich, wie der Einsatz nach Ihrem Algorithmus ablaufen müsste.

\section{Sicherheit an der Einsatzstelle}

Das Team zeigt im oben beschriebenen Fall eine umsichtige Vorgehensweise. Bereits auf der Anfahrt zum Einsatzort nutzt die Beifahrerin die Zeit, um sich ein zusätzliches Paar Einmalhandschuhe überzuziehen. Insbesondere wenn mit einer verstärkten Infektionsgefahr oder mit stark blutenden Wunden zu rechnen ist, ist dies angezeigt. Sollte das obere Paar Handschuhe verschmutzen, lässt es sich rasch ausziehen - die darunterliegenden Handschuhe bieten aber weiterhin Schutz.

Ob das Team nach dem SSSS-Schema (S-cene, S-afety, S-ituation, S-upport), nach dem SICK-Schema (S-icherheit, I-mpression, C-ritical bleeding, K-inematik) oder einer anderen Merkhilfe vorgeht, ist dabei nicht von Belang. Wichtig ist, den Eigenschutz zu beachten und einen ersten Eindruck zu gewinnen, um Gefahren zu vermeiden.

\section{PRAXISTIPP}

Gängige Merkhilfen haben wir für Sie in dem Plakat „Fit für den Einsatz? Das sollten Sie wissen!“ zusammengefasst. Von SSSS bis zu IPAP und zur SAMPLER+SAnamnese bietet es eine gute Übersicht zur Vorbereitung auf Ihre Ergänzungsprüfung. Das Plakat können Sie kostenfrei bestellen unter www.thieme.de/rettenposter.

Die Fixerutensilien mit dem Schuh zur Seite zu schieben, birgt die Gefahr, dass sich eine Person zu einem späteren Zeitpunkt an der Nadel verletzt. In der konkreten Situation ist die Vorgehensweise sicherlich akzeptabel. Wichtig ist es aber, die Gefahrenquelle nicht zu vergessen und bei Gelegenheit zu entfernen.

\section{PRAXISTIPP}

Schützen Sie Ihr Team vor Verletzungen: Wenn Sie Gefahrenquellen nicht sofort beseitigen können, weisen Sie Ihren Kollegen darauf hin. Lassen Sie sich bestätigen, dass er die Information auch wirklich verstanden hat!

\section{Geeigneter Raum für die Versorgung}

Gleich nach dem Eintreffen entscheidet sich das Team, die Patientin in einen Bereich mit mehr Raum für die Versorgung zu bringen. Das Mitführen einer Einmaldecke gehört dabei zum kleinen Einmaleins. Besser wäre es gewesen, zusätzlich eine Rettungsdecke unter die Einmaldecke zu legen: Die Rettungsdecke würde die Patientin gegen die Kälte des Bodens isolieren und die Einmaldecke zudem die Wärmeleitung reduzieren. Aufgrund der vordringlichen Sicherung der Vitalfunktionen ist die gewählte Vorgehensweise allerdings begründbar.

\section{Sicherung der Vitalfunktionen}

Nach dem Freimachen der Atemwege entscheidet sich das Team für die assistierte Beatmung. Der Helfer am Notfallkoffer übernimmt diese Aufgabe, während die Notfallsanitäterin die Untersuchung fortführt. Je nachdem, wie sich das Team zusammensetzt, ist diese Aufgabenteilung kritisch zu diskutieren. Da die assistierte Beatmung viel Erfahrung erfordert, sollte sie nur dann delegiert werden, wenn der Kollege sie auch wirklich beherrscht. Gleichzeitig assistiert zu beatmen und den Kollegen in den Untersuchungsschritten anzuleiten, ist äußerst problematisch - das lässt sich aber bei entsprechenden Konstellationen des Teams nicht anders umsetzen. 


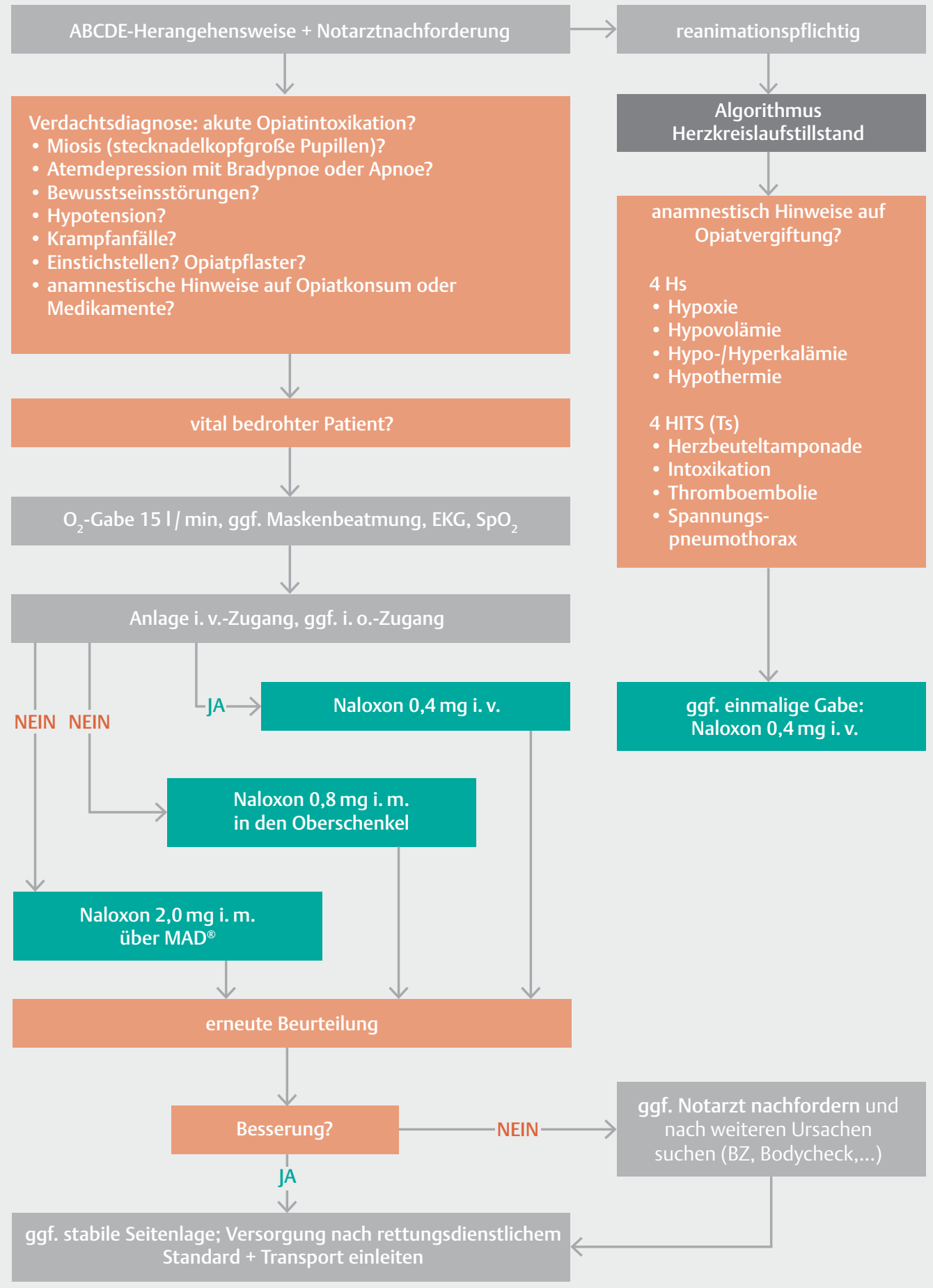

- Abb. 1 Vorgehensweise nach dem Algorithmus „Opiatintoxikation - Naloxon“ der Deutschen Angestellten-Akademie Meiningen (Stand 06/ 2015, Version 1.2). BZ = Blutzucker, $M A D^{\circledR}=$ Mucosal Atomization Device, $\mathrm{SpO}_{2}=$ pulsoxymetrisch gemessene Sauerstoffsättigung. Quelle: Deutsche Angestellten-Akademie (DAA) Meiningen. 


\section{ABCDE-Schema}

Konsequent arbeitet das Team das ABCDE-Schema ab. Gerade in Situationen, die erst einmal klar erscheinen, hilft das Schema, strukturiert vorzugehen und keine Differenzialdiagnosen zu übersehen. Insbesondere bei bewusstseinsveränderten Patienten muss man neben dem Pupillenstatus auch immer der Blutzuckerspiegel bestimmen. Die eingehende körperliche Untersuchung hilft, verborgene Verletzungen aufzuspüren (z. B. Schwellungen oder Blutungen in den Haaren, Einstichstellen etc.), und vermeidet diagnostische Fallgruben.

\section{Antidot}

Die Verdachtsdiagnose im beschriebenen Fall ist eindeutig und das Team entschließt sich zur Gabe von $0,3 \mathrm{mg}$ Naloxon i. v. Unserer Einschätzung nach fehlt im Algorithmus der Hinweis zur langsamen und fraktionierten Gabe von Naloxon. Das Team arbeitet vorbildlich und verdünnt das Antidot auf $8 \mathrm{ml}$, dadurch wird die langsame Applikation deutlich einfacher. Sobald die Patientin wieder ausreichend spontan atmet, verzichtet das Team auf eine weitere Naloxon-Gabe.

\section{PRAXISTIPP}

Sie müssen Medikamente über längere Zeit langsam verabreichen? So geht's einfacher: Verdünnen Sie das Medikament, falls möglich, auf eine leichter zu applizierende Menge oder verabreichen Sie es über die Infusion oder eine Spritzenpumpe.

\section{Prüfungsfragen}

\section{NOTFALLMEDIZIN}

? Warum ist die Gabe von Naloxon bei Opiatintoxikationen nicht unproblematisch? Beschreiben Sie grob die physiologischen Wirkungsweisen von Opiaten und dem Antidot Naloxon.

Naloxon ist ein kompetitiver Antagonist, der mit dem Opiat - in diesem Fall aller Wahrscheinlichkeit nach Heroin - um die Opioid-Rezeptoren konkurriert. Naloxon verfügt über eine höhere Affinität zu den Rezeptoren als Opiate und hebt deren Wirkung deshalb zeitweise auf. Da Naloxon nur eine relativ geringe Wirkdauer von etwa $2 \mathrm{~h}$ hat, wirkt es deutlich kürzer als die meisten Opiate. Die Folge ist ein sog. Rebound-Effekt: Die Symptome werden vorübergehend besser, können aber nach einiger Zeit wieder auftreten. Dies ist insbesondere dann problematisch, wenn der Patient nach der Gabe von Naloxon wieder zu Bewusstsein kommt und die weitere Therapie und Versorgung verweigert. Gerade bei Patienten mit einer Opiatabhängigkeit kann die rasche Gabe und Überdosierung des Antidots zu plötzlichen Entzugserscheinungen führen. Nicht selten eskaliert dann die Lage - z. B. indem sich der Patient der Behandlung entzieht und flüchtet.

\section{Mögliche weitere Fragen:}

- Welche Möglichkeiten zur Applikation von Naloxon haben Sie, wenn der i. v.-Weg nicht möglich ist? Beschreiben Sie Ihre Vorgehensweise und die damit verbundenen Schwierigkeiten.

- Nehmen Sie Stellung zur drohenden Hypothermie der Patientin. Welche Faktoren wirken sich dabei begünstigend aus?

\section{KOMMUNIKATION}

? In dem beschriebenen Fall kommt es zu einer Konfliktsituation mit dem vermeintlichen Freund der Patientin. Versetzen Sie sich in die Situation des jungen Manns und erörtern Sie, welche Reaktionen auf den versuchten Diebstahl angemessen wären.

Der junge Mann in der beschriebenen Situation ist wahrscheinlich auch opiatabhängig. Er wirkt agitiert, wird zunehmend unruhig, zittert und schwitzt. Die Symptome deuten auf eine eskalierende Entzugssymptomatik hin. In dieser Situation steht die Befriedigung des Suchtbedürfnisses im Fokus des Betroffenen. Der Griff in das Ampullarium erscheint in dieser Lage als ein geeigneter Ausweg. Die Frage, ob man im Rettungsdienst den nicht vollzogenen Diebstahl zur Anzeige bringen sollte, erübrigt sich: Der junge Mann ist aufgrund seiner Abhängigkeit sicherlich nur bedingt schuldfähig; außerdem ist noch kein Schaden entstanden. Würde er sich durch eine klare Anweisung nicht vom Diebstahl abbringen lassen, wäre es ratsam, die Polizei zeitnah hinzuzuziehen. Eine Eskalation im Sinne einer körperlichen Auseinandersetzung sollte man möglichst umgehen. Die Gefahr einer Verletzung ist zu groß und die Versorgung der Patientin würde leiden.

\section{Mögliche weitere Fragen:}

- Worauf müssen Sie im Umgang mit der oben beschriebenen Zielgruppe besonders achten?

- Was ist bei der Kommunikation mit Drogenabhängigen zu bedenken?

\section{RAHMENBEDINGUNGEN}

? Der junge Mann versucht sich gewaltsam Zugriff auf Ihr Ampullarium zu verschaffen. Es kommt zum Handgemenge. Beschreiben Sie, welche Maßnahmen Ihnen im Rahmen dieser Auseinandersetzung zugebilligt werden. Auf welche gesetzliche Regelung beziehen Sie sich?

In diesem Fall möchte sich das Rettungsdienstpersonal vor dem gewaltsamen Zugriff auf das Ampullarium schützen. Es handelt zu Anfang wohl im Sinne eines rechtfertigenden Notstands gemäß §34 StGB, bei dem der Täter Individualrechtsgüter - wie z. B. das Eigentum 
- schützen möchte und deshalb nicht rechtswidrig handelt. Dabei geht es also um eine Rechtsgüterabwägung. Erst im Rahmen der tätlichen Auseinandersetzung kommt es zur Notwehr ( $\$ 32$ StGB). Dieser Paragraf besagt: Wer einen rechtswidrigen Angriff gegen sich selbst oder einen anderen abwehrt, macht sich nicht strafbar. Bei der Wahl der einzusetzenden Mittel muss man dabei auf die Verhältnismäßigkeit achten. Anzumerken ist, dass Rettungsdienstpersonal nicht die exekutive Gewalt im Staat ersetzt oder ergänzt: Im Zweifel müssen Sie einen
Täter mit seiner Beute ziehen lassen und den Sachverhalt an die Polizei weitergeben .

\section{Mögliche weitere Fragen:}

- Der Polizeibeamte möchte von der Notfallsanitäterin Auskünfte zur Verdachtsdiagnose erhalten. Nehmen Sie Stellung zur rechtlichen Problematik.

- Welche besonderen Infektionsgefahren bestehen für Sie im Zusammenhang mit der oben beschriebenen Zielgruppe? Beschreiben Sie, wie Sie sich schützen können.

\section{KOMMENTAR}

von Rico Kuhnke, Schulleiter der DRK-Landesschule Baden-Württemberg und Mitherausgeber von retten!

Am 12. September 2016 hat der wissenschaftliche Dienst des deutschen Bundestags eine Ausarbeitung zu folgenden Fragen veröffentlicht: Wie ist die Ausbildungszielbestimmung des Notfallsanitätergesetzes (NotSanG) im §4 Abs. 2 Nr. 2c zu interpretieren? Und welche Absicht verfolgte der Gesetzgeber damit? Der 51 Seiten umfassende Text beschreibt die Rahmenbedingungen, unter denen der Notfallsanitäter in der Praxis tätig wird [1]. Insbesondere geht es dabei um Maßnahmen, die nach § 1 Abs. 1 Heilpraktikergesetz (HeilprG) unter dem sog. Arztvorbehalt stehen.

Das HeilprG vom 17. Februar 1939 ist nach wie vor die Grundlage dafür, dass die Ausübung der Heilkunde nur Ärzten gestattet ist - andere Personen bedürfen dafür einer besonderen Erlaubnis. Unter strengen Auflagen sind Heilpraktiker und Hebammen von dieser Regelung ausgenommen - Notfallsanitäter aber nicht. Ein Notfallsanitäter verstößt im Rahmen seiner Berufsausübung gegen die Einschränkungen des HeilprG, wenn er eigenständig ärztliche Maßnahmen durchführt.

In NotSanG § 4 regelt der Gesetzgeber das Ausbildungsziel. Danach soll ein Notfallsanitäter befähigt werden, bestimmte Aufgaben eigenverantwortlich, andere im Rahmen der Mitwirkung auszuführen. Diese Maßnahmen sind in Abs. 2 Nr. 1 bzw. Abs. 2 Nr. 2 detailliert aufgeführt.

- Die eigenverantwortlich zu erledigenden Aufgaben umfassen auch Notfallsituationen, in denen der Notfallsanitäter bis zur Übernahme durch einen Arzt zeitlich befristet heilkundlich tätig wird; sie sind unter § 4 Abs. 2 Nr. 1 Buchstabe c beschrieben. Dort heißt es: „[...] Anwenden von in der Ausbildung erlernten und beherrschten, auch invasiven Maßnahmen, um einer Verschlechterung der Situation [...] vorzubeugen, wenn ein lebensgefährlicher Zustand vorliegt oder wesentliche Folgeschäden zu erwarten sind. “ Das bedeutet: Der Notfallsanitäter nimmt im konkreten Fall eine Güterabwägung vor und entscheidet sich entgegen der Einschränkung des HeilprG, heilkundlich tätig zu werden. Damit beruft er sich auf den vielzitierten $§ 34$ StGB, den rechtfertigenden Notstand. Diese Argumentationskette unterscheidet sich also nicht von der Situation der Rettungsassistenten in der Vergangenheit. Lediglich in einer Einzelfallbewertung bei einer juristischen Auseinandersetzung kann der Notfallsanitäter darauf verweisen, dass der Gesetzgeber die notwendige Durchführung von erweiterten Maßnahmen für einen Notfallsanitäter grundsätzlich erkannt hat.

- Spannend ist, wie der wissenschaftliche Dienst des Bundestags die Maßnahmen bewertet, die der Notfallsanitäter im Rahmen der Mitwirkung ausüben soll. Laut § 4 Abs. 2 Nr. 2 Buchstabe c gehört dazu u. a. das „... eigenständige Durchführen von heilkundlichen Maßnahmen, die vom ärztlichen Leiter Rettungsdienst [...] bei bestimmten notfallmedizinischen Zustandsbildern und -situationen standardmäßig vorgegeben, überprüft und verantwortet werden. “ Der wissenschaftliche Dienst schreibt, der ÄLRD könne dies grundsätzlich über standardisierte Handlungsanweisungen - die sog. SOP (Standard Operating Procedures) oder Algorithmen - umsetzen. Allerdings sei von einer Delegation nach allgemeinem juristischem Verständnis nur dann auszugehen, wenn eine SOP möglichst präzise formuliert ist und keinen Bewertungsspielraum für den Notfallsanitäter enthält. Ist dies nicht der Fall, müsste der Notfallsanitäter eine ärztliche Behandlungsentscheidung treffen und nach den Regeln einer Substitution tätig werden. Bei der Substitution übernimmt der Notfallsanitäter ärztliche Maßnahmen und die damit verbundene Entscheidungshoheit darüber, ob eine Maßnahme indiziert ist oder nicht. Der wissenschaftliche Dienst betont, dass eine Substitution ärztlicher Behandlungsentscheidungen nicht erkennbarer Wille des Gesetzgebers war. Wäre dies das Ziel gewesen, hätte er das HeilprG ändern müssen. 
FAZIT

Nach wie vor gibt es Situationen, in denen der Notfallsanitäter invasive Maßnahmen ergreift und dabei gegen das HeilprG verstößt. Dabei beruft er sich, wie der Rettungsassistent, auf den rechtfertigenden Notstand. Dort, wo ein verantwortlicher Arzt Algorithmen für bestimmte notfallmedizinische Notfallbilder vorgibt und überprüft, werden Notfallsanitäter im Sinne einer Delegation tätig. Die Algorithmen müssen allerdings so eng gefasst sein, dass möglichst kein Entscheidungsspielraum für den Notfallsanitäter besteht. In den kommenden Jahren ist es Aufgabe der Länder, die Berufsausübung von Notfallsanitätern durch geeignete Rahmenvorgaben zu regeln. Meines Erachtens wäre es zielführender gewesen, das HeilprG zu reformieren und darin dem Notfallsanitäter eine eigene notfallmedizinische Profession mit einem fest umrissenen Handlungsrahmen einzuräumen - denn bei Einführung des HeilprG vor > 85 Jahren gab es die präklinische Notfallmedizin, wie wir sie heute kennen, noch nicht.

\section{Interessenkonflikt}

Die Autoren geben an, dass kein Interessenkonflikt besteht.

Über die Autoren

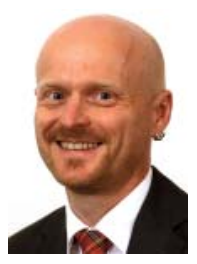

\section{Rico Kuhnke}

ist Schulleiter der DRK-Landesschule BadenWürttemberg. Er war viele Jahre als Lehrrettungsassistent tätig und ist Notfallsanitäter. Er ist Mitherausgeber von retten!.

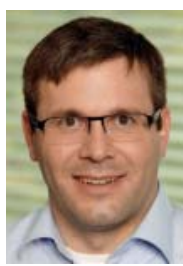

\section{Dr. med. Wolfgang C. G. von Meißner}

war bis Ende 2015 Ärztlicher Leiter der DRKLandesschule Baden-Württemberg und ist ERCALS Course Director. Er ist Facharzt für Anästhesiologie mit den Zusatzbezeichnungen Notfallmedizin und Intensivmedizin. Zugleich ist Dr. von Meißner Facharzt für Allgemeinmedizin und arbeitet als Landarzt und Notarzt in Baiersbronn im Schwarzwald. Er ist Mitherausgeber von retten!.
Korrespondenzadresse

\section{Rico Kuhnke}

Schulleiter

Deutsches Rotes Kreuz

Landesschule Baden-Württemberg

Karl-Berner-Str. 6

72285 Pfalzgrafenweiler

r.kuhnke@drk-Is.de

\section{Literatur}

[1] Wissenschaftliche Dienste des Deutschen Bundestags. Ausarbeitung WD 9 - 3000 - 042/16. Die Ausbildungszielbestimmung des $\S 4$ Abs. 2 Nr. 2 Buchstabe c des Notfallsanitätergesetzes - Bundesrechtliche Vorgaben und Umsetzung durch die Bundesländer (12.09.2016). Im Internet: https://www. bundestag.de/blob/476080/0c5c298bbbe9e7b9c0ea67f1 61c0a190/wd-9-042-16-pdf-data.pdf Stand: 15.12.2016

\section{Bibliografie}

DOI http://dx.doi.org/10.1055/s-0042-118152 | retten 2017; 6: 18-24

(c) Georg Thieme Verlag KG Stuttgart · New York ISSN 2193-2387 